\title{
Las Tecnologías de la Información como un factor de mejora en el proceso de enseñanza-aprendizaje en la educación a distancia en una universidad
}

\author{
Cecilia García Muñoz Aparicio, Universidad Juárez Autónoma de Tabasco, México \\ José Manuel Piña Gutiérrez, Universidad Juárez Autónoma de Tabasco, México \\ María del Carmen Ancona Alcocer, Universidad Juárez Autónoma de Tabasco, México \\ María del Carmen Navarrete Torres, Universidad Juárez Autónoma de Tabasco, México
}

\begin{abstract}
Resumen: El presente artículo muestra el impacto que han tenido las nuevas tecnologías, llámense de la información y/o de la comunicación en la Universidad Juárez Autónoma de Tabasco [UJAT], las cuales han revolucionado la manera de percibir, acceder y transmitir el conocimiento conformando día con día una parte en el proceso de enseñanza-aprendizaje; así como su utilización en las aulas escolares, tanto de estudiantes como profesores, debido a que ambos se benefician en la utilización de las TIC en la educación, ya que se estimula el aprendizaje de manera continua, siendo parte de la cultura actual para el desarrollo de la Universidades, que han puesto en marcha programas de educación a distancia para poder llegar a todos los lugares. La metodología utilizada es de tipo documental, teniendo como objetivo mostrar los avances de la universidad.
\end{abstract}

Palabras clave: Universidad Juárez Autónoma de Tabasco, proceso enseñanza-aprendizaje, TIC

\begin{abstract}
This article shows the impact that new technologies have had, whether they be information and / or communication in Universidad Juárez Autónoma de Tabasco [UJAT], which have revolutionized the way we perceive, access and transmit knowledge conforming day by day with a part in the teaching-learning process as well as its use in the classroom, both students and teachers, because they both benefit in the use of ICTs in education, and that learning is stimulated continuously, being part of today's culture for the development of the universities that have launched distance education programs to reach all places. The methodology used is documentary, aiming to show the progress of the university.
\end{abstract}

Keywords: Universidad Juárez Autónoma de Tabasco, Teaching-Learning, ICT

\section{Introducción}

$\mathrm{L}$

a brecha digital es uno de los primeros conceptos con que se inicia la reflexión alrededor del tema del impacto social de las tecnologías de información y comunicación (TIC), de acuerdo con Tello (2007) y desde entonces se va percibiendo que estas tecnologías van a producir diferencias en las oportunidades de desarrollo de las poblaciones y que se establecerá una distancia entre las que tienen y las que no tienen acceso a las mismas. Las TIC es un término que contempla toda forma de tecnología utilizada para crear, almacenar, intercambiar y procesar información en sus varias formas, tales como datos, conversaciones de voz, imágenes fijas o en movimiento, presentaciones formas, incluyendo aquéllas aún no concebidas. En particular, están íntimamente relacionadas con computadoras, software y telecomunicaciones. El problema de su acceso no sólo implica una brecha digital entre México y otros países, sino un rezago digital al interior del país, marcado por una desigualdad en el desarrollo de las mismas en la sociedad. La evidencia indica que las brechas digitales se deben a diferencias culturales, de edad e ingresos, entre otros.

La utilización de las TIC, así como su combinación con otras formas de comunicación social, incluye también la eventualidad de producir contenidos propios, o bien, de acceder a contenidos de otros que resulten útiles, o sea es la armonización adecuada del recurso Internet con otros, como son la radio comunitaria, las reuniones presenciales, los materiales impresos y los videos, deben aprovecharse para el desarrollo integral de una comunidad, es decir tener una visión integral de desarrollo

Revista Internacional de Tecnología, Ciencia y Sociedad

Volumen 4, Número 1, <http://tecnociencia-sociedad.com>, ISSN 2530-4895

(C) Global Knowledge Academics. Cecilia García Muñoz Aparicio et al.

Todos los Derechos Reservados Permisos: soporte@gkacademics.com

Republicado de Revista Internacional de Tecnología, Conocimiento y Sociedad 4(1), 2015 (pp. 85-94) 
apuntando hacia el crecimiento económico sino, que se impulse el potencial humano en sus diferentes dimensiones para afianzar así la prosperidad económica, con equidad, y el fortalecimiento democrático con transparencia y justicia social (Sánchez, 2008).

La transformación producida por las TIC no sólo ha cambiado la cultura y los medios de comunicación masivos si no también ha tenido un impacto irreversible en el mundo del trabajo. Este hecho involucra especialmente a la educación superior, ya que en la actualidad se necesitan personas alfabetizadas en el uso de estas tecnologías y educadas a lo largo de la vida (Salas, 2005).

Actualmente muchas universidades de diferentes países, han puesto en marcha programas de introducción de las TIC en los procesos de enseñanza y aprendizaje, y estos programas han venido acompañados de propuestas de formación para el profesorado. Estos procesos también se han tenido que adaptar en gran medida al crecimiento en la oferta de programas educativos virtuales, cuyo principal soporte son los medios didácticos interactivos, y que conforme se han dado mayores avances, se ha incrementado la oferta de diversos programas y cursos con actividades totalmente en línea o mixtas, es decir presenciales y en línea.

Las TIC han tenido su origen en los mismos ambientes universitarios y, por lo mismo, han sido las más utilizadas, independientemente de que su uso se ha extendido a todos los niveles educativos y a diversas áreas del quehacer humano y quizás el desarrollo más conocido y estudiado es que se da en el contexto universitario a través de la modalidad de educación a distancia (Martínez y Heredia, 2010).

Alvarado y Dorrego (2003) afirman que la incorporación de las TIC a veces van acompañadas de procesos de investigación sobre su pertinencia en el ámbito educativo, y de la capacitación requerida, no sólo de los profesores, sino también del personal técnico administrativo que deberá participar en la producción y administración de los cursos a distancia.

Los entornos virtuales de enseñanza aprendizaje pueden ser considerados como un tipo especial de espacio o situación, en el que se realiza un proceso de enseñanza-aprendizaje en línea, caracterizado por el predominio de la separación física entre profesores y estudiantes, el estudio independiente y una comunicación mediada por las tecnologías.

Edel-Navarro (2010) comenta que la tecnología tiene muchos retos que cumplir en la educación la cual consiste en la evolución de las formas de concebir, actuar, comprender, actuar, implementar las acciones educativas en el contexto social y aunque el estudio de las TIC y su relación con la educación representan vertientes relevantes de conocimiento sobre los entornos virtuales de aprendizaje, debido a que en las últimas décadas la investigación científica sobre el proceso de aprendizaje y su relación con la tecnología ha generado seis líneas de conocimiento frontera sobre entornos virtuales de aprendizaje, lo cuales son: el desarrollo de la tecnología educativa, el empleo de las TIC en el proceso educativo, el impacto de las plataformas tecnológicas en la educación, la influencia del internet en los procesos educativos, los modelos y modalidades de educación a distancia y el fenómeno de la virtualización educativa.

De acuerdo con el autor (Edel-Navarro, 2010) es importante mencionar en los entornos virtuales, la participación de Bustos y Coll de laUniversidad de Barcelona, ya que desde un enfoque constructivista de orientación sociocultural presentan un modelo teórico que analiza la transformación potencial de los entornos virtuales a partir de la capacidad de mediar las relaciones entre profesores, estudiantes y contenidos.

La idea de un proyecto educativo virtual no se diferencia de los demás sistemas de educación ya que se incluyen profesores y estudiantes en los cuales se da la apropiación del conocimiento así como contenidos de aprendizaje a través de un marco institucional en el cual se generan modelos de trabajo y de vinculación entre personas y contenidos ( Torres, 2005). De igual forma, Torres (2005) afirma que la educación virtual o electrónica es el desarrollo de programas académicos a distancia soportados con tecnología a través de redes teleinformáticas.

Los entornos virtuales de enseñanza-aprendizaje es un concepto que viene asociado a un nuevo paradigma en el cual convergen las tendencias actuales de la educación, para todo tipo de estudiante y durante toda la vida, en el cual las teorías y estilos de aprendizaje centran sus procesos en el estudiante, lo que permite construir un conocimiento basado en sus propias expectativas y necesidades de acuerdo al contexto en que se desarrolla, ya sea aplicando métodos investigativos que le permitan 
tomar acciones para alcanzar resultados positivos; de manera unida y fácil por el uso intensivo de las TIC y el trabajo en red, cuyo mayor reto es mantener y elevar la calidad del proceso docenteeducativo en donde la enseñanza presencial está siendo desplazada por métodos de enseñanza más flexibles, en los cuales no es necesario el contacto físico profesor-estudiante, salvo algunas reuniones periódicas indispensables (Vidal, Llanusa, Diego, y Vialart, 2008).

Este artículo presenta una investigación exploratoria y documental con un enfoque cuantitativo y un alcance exploratorio, utilizando fuentes primarias y secundarias en la cual se destacan los logros en la implementación de la Educación a Distancia en la Universidad Juárez Autónoma de Tabasco.

\section{Tecnología educativa}

La Tecnología Educativa según Salas (2002), abre una serie de problemáticas completamente nuevas, que involucran lo específicamente tecnológico; esto es, lo que tiene que ver con los medios de comunicación, en tanto dispositivos, así como sus posibilidades comunicacionales, y en sí misma no puede garantizar el convertirse en una herramienta eficiente en el ámbito educativo, sino que ello va a depender del paradigma educativo y de la pedagogía que se elijan, tanto como de la manera en que sea implementada en el ámbito didáctico.

De acuerdo con Vidal, Nolla, y Diego (2009) la tecnología educativa es el "acercamiento científico basado en la teoría de sistemas que proporciona al educador las herramientas de planeación y desarrollo, así como la tecnología que busca mejorar el proceso de enseñanza-aprendizaje a través del logro de los objetivos de enseñanza son educativos y buscando la efectividad del aprendizaje", por lo que las plataformas didácticas son sistemas que son vistos como dispositivos que contribuyen para lograr el aprendizaje y que utilizan modelos pedagógicos de enseñanza y aprendizaje respectivamente.

Se puede decir que la tecnología educativa es la complejidad del tema pues un texto resulta insuficiente para abordar con profundidad los distintos aspectos de la relación entre educación y tecnología quedando abierta la posibilidad de seguir trabajando sobre temas como: alfabetización digital y didáctica, recursos tecnológicos dentro y fuera del aula, análisis, selección, utilización, diseño, producción, evaluación e investigación de medios, en los procesos de aprendizaje-enseñanza, lo cual trae como consecuencia la formación de la educación a distancia, en un afán de por poder abarcar un mayor número de estudiantes en las universidades teniendo como finalidad la calidad educativa (Ortega, 2010), otro punto importante del autor es que la sociedad del conocimiento demanda un salto cualitativo en los sistemas educativos; requiere avanzar en la nueva alfabetización digital y desarrollar habilidades transversales como la creatividad, innovación, colaboración, capacidad de comunicación, pensamiento crítico, y así poder ayudar a los ciudadanos del siglo xxi a convertirse en seres mejor formados, aptos para desenvolverse en la sociedad del conocimiento.

Las plataformas didácticas tecnológicas más utilizadas de acuerdo con Vidal, Nolla, y Diego (2009:139) son: a) herramientas de comunicación: foros, chats, correo electrónico, b) herramientas de los estudiantes: autoevaluaciones, zonas de trabajo en grupo, perfiles, c) herramientas de productividad: calendario, marcadores, ayuda, d) herramientas de administración: autorización, administración, y e) herramientas del curso: anuncios, evaluaciones.

Este tipo de plataformas son muy útiles debido a que ayudan a la hora de impartir una asignatura o curso a distancia, porque le permite al docente estar en contacto directo con los estudiantes y a éstos los ayuda a poder cumplir en tiempo y forma con sus asignaciones y todo tipo de obligaciones que tengan que realizar en las sesiones a distancia y puede ser una herramienta esencial para los docentes que imparten clase de forma presencial.

Alvarado y Dorrego (2003:74) afirman que la tecnología educativa no ha cedido espacios, por el contrario, se percibe una evolución del área, que se ha visto marcada por el auge y desarrollo de los avances de las teorías de instrucción y de aprendizaje y de los medios de comunicación.

El impacto producido por la tecnología según Piscitelli (2005), solo se sentirá cuando cambien simultáneamente (antes y/o después) en una cascada de relaciones muchas veces difíciles de prever otras prácticas y relaciones educacionales encadenadas. La capacidad de transformación no es algo 
intrínseco a la tecnología, imaginar que lo es el sueño (o la pesadilla) tecnocrática del fundamentalismo digital, y frente a ella hay que plantearse firme e inteligentemente.

Por otro lado, Los medios de enseñanza y recursos del aprendizaje, considerando como "medios" aquellos que han sido diseñados para ser utilizados en los procesos educativos y como "recursos" aquellos diseñados con otros propósitos, son adaptados por los docentes para los procesos educativos, desde hace muchos años y más recientemente la tecnología educativa, ha servido de apoyo para aumentar la efectividad del trabajo del profesor, sin llegar a sustituir su función educativa y humana, así como organizar la carga de trabajo de los estudiantes y el tiempo necesario para su formación científica, y para elevar la motivación hacia la enseñanza y el aprendizaje, y garantizar la asimilación de lo esencial educativa, (Vidal, y Del Pozo, 2008). La tecnología educativa es el resultado de las aplicaciones de diferentes concepciones y teorías educativas para la resolución de un amplio espectro de problemas y situaciones referidos a la enseñanza y al aprendizaje. Surge como disciplina en Estados Unidos de América en la década de los cincuenta del siglo pasado y ha transitado por diferentes enfoques o tendencias como enseñanza audiovisual, enseñanza programada, tecnología instruccional y diseño curricular, entre otros. Utiliza los medios y recursos de la enseñanza como componentes activos en todo proceso dirigido al desarrollo de aprendizajes

En cuanto a la utilización de las nuevas tecnologías, López (2008-1, citando a Ogalde y González, 2008) que existen varios tipos como son los tutoriales, caracterizados por conducir y guiar al usuario en la adquisición primera de cualquier tipo de conocimiento, juegos educativos, cuya utilidad se enfoca en la práctica de información, como ambientes de aprendizaje o para incorporar el aprendizaje en ciertos terrenos, las herramientas y entornos abiertos, es decir, los programas que los estudiantes combinan las páginas o sitios web, el correo electrónico, el servicios de mensajería instantánea, discos compactos, fotografías, audio y video digital, procesadores de textos, videoconferencias, bases de datos, entre otros con otros medios como Aprendizaje basado en web, mapas mentales, Prácticas de campo virtuales y Videoconferencias o las actividades para obtener un objetivo académico. De esta manera se puede afirmar que esta extensa tonalidad facilita el proceso de enseñanza-aprendizaje, no obstante, para lograr este objetivo se proponen tres reflexiones significativas: fundamentar todo proceso que involucre el uso de las nuevas tecnologías en el contexto educativo a través de: planear, analizar y diseñar los contenidos, la forma y el medio de presentación, en donde se enfatiza la aplicación de las teorías del aprendizaje y la comunicación; la consideración de la aplicación de las nuevas tecnologías en el ámbito educativo por sí sola no garantiza el proceso educativo, paradójicamente, ya que también lo puede entorpecer y pasando por alto la necesidad de diversificar e intercalar la aplicación consciente de las nuevas tecnologías con los procesos tradicionales de enseñanza-aprendizaje, se evitará el abuso de las mismas .para que impacten en el aprendizaje (López, 2008).

\section{Antecedentes del Sistema de Educación a Distancia en la UJAT}

De las 11 Divisiones Académicas que conforman a la UJAT es en el año 2003 cuando surge por primera vez la Educación a Distancia en la División Académica de Ciencias Sociales y Humanidades y es en el 2004 (ver tabla 1) su inicio en la División Académica de Ciencias Económico Administrativas y así posteriormente se van anexando otras Divisiones; la Educación a Distancia en la universidad, es la instancia administrativa que integra y coordina el trabajo de los actores que intervienen en el sistema con la finalidad de gestionar, otorgar y asegurar la existencia de los recursos materiales, tecnológicos y humanos que contribuyan al logro de los objetivos institucionalmente establecidos para la modalidad. El inicio de la educación a distancia en estas carreras es debido a la gran demanda por parte de los estudiantes, ya que en las aulas ya no caben más, Actualmente se cuenta con 527 estudiantes en esta modalidad.

El Modelo Institucional está orientado fundamentalmente por el Modelo Educativo de la Universidad Juárez Autónoma de Tabasco, (ver la tabla 2), basado en la concepción pedagógica constructivista y humanista del aprendizaje, centrándose perspectiva su estrategia educativa se centra en el estudiante, ofreciendo una alternativa de formación en educación superior a las personas que 
trabajan y desean cursar o concluir una carrera profesional sin tener que separarse de su actividad laboral, por lo que la educación a distancia se define como una modalidad educativa caracterizada por la responsabilidad de enseñar, generar, aplicar conocimientos y construir aprendizaje haciendo uso de las tecnologías de la información y la comunicación para mediar el diálogo entre estudiantes, profesores separados en tiempo y espacio, potenciando un aprendizaje independiente y flexible (UJAT, 2013b).

Tabla 1: Divisiones Académicas con carreras a distancia y número de estudiantes

\begin{tabular}{|c|c|c|c|c|c|c|c|}
\hline $\begin{array}{c}\text { División } \\
\text { Cienciámica de }\end{array}$ & $\begin{array}{c}N^{o} \\
\text { alumnos } \\
\text { Económico }\end{array}$ & $\begin{array}{c}\text { División } \\
\text { Académica } \\
\text { de Educa- } \\
\text { ción y Artes }\end{array}$ & $\begin{array}{c}N^{o} \\
\text { alumnos }\end{array}$ & $\begin{array}{c}\text { División } \\
\text { Académica } \\
\text { de Ciencias } \\
\text { de la Salud }\end{array}$ & $\begin{array}{c}N^{o} \\
\text { alumnos }\end{array}$ & $\begin{array}{c}\text { División } \\
\text { Académica } \\
\text { de Ciencias } \\
\text { Sociales y } \\
\text { Humanidades }\end{array}$ & $\begin{array}{c}N^{o} \\
\text { alumnos }\end{array}$ \\
$\begin{array}{c}\text { Lic. En Ad- } \\
\text { ministración }\end{array}$ & 106 & $\begin{array}{c}\text { Maestría de } \\
\text { enseñanza } \\
\text { del idioma } \\
\text { inglés }\end{array}$ & 4 & $\begin{array}{c}\text { Curso com- } \\
\text { plementario } \\
\text { de la Licen- } \\
\text { ciatura en } \\
\text { Enfermería }\end{array}$ & 58 & $\begin{array}{c}\text { Licenciatura } \\
\text { en Derecho }\end{array}$ & 252 \\
\hline $\begin{array}{c}\text { Lic. En Con- } \\
\text { tabilidad }\end{array}$ & 63 & $\begin{array}{c}\text { Licenciatura } \\
\text { en Desarrollo } \\
\text { Cultural } \\
\text { (semipresen- } \\
\text { cial) }\end{array}$ & 44 & & 58 & & 252 \\
\hline $\begin{array}{c}\text { Total de } \\
\text { estudiantes }\end{array}$ & 169 & 48 & & & \\
\hline
\end{tabular}

Fuente: Elaboración propia basada en UJATa (2013), Segundo Informe de actividades.

Tabla 2: Características de los estudiantes a distancia en la UJAT

\begin{tabular}{|l|}
\hline El estudiante desarrolla su aprendizaje bajo la orientación del docente tutor. \\
\hline Se combinan estrategias, métodos y medios técnicos y didácticos. \\
\hline Se aprende con la orientación de guías didácticas posibilitando la autoevaluación. \\
\hline $\begin{array}{l}\text { Existe capacidad de aprender más allá de un aula, eliminando las barreras de tiempo y espacio } \\
\text { por lo que no implica la asistencia a clases }\end{array}$ \\
\hline Garantiza al estudiante la misma calidad de aprendizaje que en la modalidad escolarizada \\
\hline
\end{tabular}

Fuente: Elaboración propia basado en http://www.test.ujat.mx/interioradentro.aspx? ID =15379

$\& N O D O=229$

\section{Modalidades del proceso de enseñanza-aprendizaje}

En la Universidad Juárez Autónoma de Tabasco existen 3 tipos de proceso de enseñanzaaprendizaje: presencial, a distancia y semipresencial, en los cuales se destacan diferencias en cuanto al contenido y la orientación didáctica debido a que el público meta que son los estudiantes es diferente (UJATb, 2013) . En el área a distancia los estudiantes poseen un mayor sentido de responsabilidad, una disposición de innovar, son autodidactas, autogestivos y tienen un mayor compromiso a la hora de estudiar los materiales didácticos así como amplios conocimientos en computación.

En este año en la UJAT se acaban de abrir Centros de Educación a distancia en municipios lejanos del estado de Tabasco como son Jonuta y Jalapa para que la educación llegue a más puntos importantes, teniendo la educación semipresencial, (ver tabla 3) impartiéndose las carreras de Licenciado en Administración, Licenciado en Contaduría pública, y Licenciatura en Trabajo Social con un total de 81 estudiantes. Estos municipios están rezagados y la universidad al no poseer infraestructura suficiente para hacer más Divisiones Académicas, significa una oportunidad de enseñar a un número mayor de estudiantes ya que el crecimiento de la población demanda más educación para sus estudiantes. 
Tabla 3: Número de estudiantes semipresenciales en Jalapa y Jonuta

\begin{tabular}{|l|c|l|c|}
\hline Jalapa & $N^{o}$ de Estudiantes & Jonuta & $N^{o}$ de estudiantes \\
\hline Licenciado en Administración & 8 & Licenciado en Administración & 5 \\
\hline $\begin{array}{l}\text { Licenciado en Contaduría } \\
\text { Pública }\end{array}$ & 41 & $\begin{array}{l}\text { Licenciado en Contaduría } \\
\text { Pública }\end{array}$ & 3 \\
\hline Licenciatura en Trabajo social & 16 & Licenciatura en Trabajo social & 8 \\
\hline
\end{tabular}

Fuente: Elaboración propia, (UJAT, 2014) en Servicios Escolares.

El proceso de enseñanza es consciente por su naturaleza social, lo que significa que el estudiante se compromete para su enseñanza pues tiene al hombre en su centro y busca el desarrollo, el compromiso y la responsabilidad del sujeto en su propio proceso de formación. Su carácter holistico se expresa en su naturaleza totalizadora, porque cada una de las partes que forman parte del proceso, como objeto de estudio, está en constante interacción con las restantes, lo que hace que en cada una de estas se reflejen las cualidades del objeto como un todo, a la vez que en el todo se refleja el significado de cada una de las partes (Mestre, y Valdés, 2010). Esto es que el alumno a distancia tendrá que formarse igual que uno de forma presencial con las mismas características y conocimientos y aunque los contenidos no son idénticos, los estudiantes al salir tienen que saber lo mismo y al menos poseer competencias generales.

En un proceso educativo de tipo virtual se busca que la secuencia didáctica multimedia tenga no solo el orden de presentación de los contenidos de aprendizaje, sino que además, debe tener del discurso del docente, mediatizado por algún tipo de recurso tecnológico. Esto va dirigido a suplir la orientación verbal que normalmente se da de manera frontal en la enseñanza presencial, por lo cual se debe de estimular la activación de los esquemas mentales en función de los conocimientos previos para crear las condiciones que le permitan al estudiante asimilar con mayor facilidad los contenidos que debe aprender.

Un factor de significativo valor en todo este proceso de introducción de las TIC en la enseñanza, lo constituye la participación del docente en la elaboración de los materiales instructivos. Esto constituye un tema muy polémico dentro del mundo académico en la actualidad, sobresaliendo tres enfoques fundamentales al respecto (Mestre y Valdés, 2010, p.39): el primero parte de que debe ser el profesor el encargado de producir sus materiales, por lo tanto, esto le exige estar la día en el uso de las tecnologías y una actualización continua para poder conocer las más recientes herramientas informáticas en aras de poder utilizar las más idóneas a la hora de resolver un problema; el segundo plantea que es tarea de los especialistas en las ciencias de la computación producir los materiales educativos que necesitan los profesores y para ello deben apropiarse de los conocimientos pedagógicos y psicológicos necesarios para emprender esa tarea y el tercero se argumenta que el éxito se logra con la creación de grupos de trabajo multidisciplinarios en el que intervengan pedagogos, informáticos, psicólogos, entre otros, ya que es esta la única forma de obtener productos completos y de alta calidad.

De acuerdo con Sancho, Ornellas, Sánchez, Alonso, y Bosco, (2008, citando a Sancho, 2006) los principales problemas identificados en la implementación de nuevas perspectivas de enseñanza y aprendizaje incorporando las TIC se centran la utilización de herramientas, el contenido, la articulación y las formas de definir los niveles de logro de los currículos actuales, que dificultan las propuestas transdisciplinares, el aprendizaje basado en problemas y la transformación de los sistemas de evaluación, las restricciones que provienen de la propia Administración, y los esquemas de organización en la enseñanza, la organización del espacio -acceso a los ordenadores, número de estudiantes por clase - y del tiempo escolar, a los sistemas de formación permanente del profesorado que impiden el cambio educativo, la falta de motivación por parte del profesorado para introducir nuevos métodos y la poca autonomía que gozan el profesorado y el alumnado.

\section{Formación docente}

La formación inicial del profesorado según Sancho et. al (2008), se configura cada vez más como un elemento clave y estratégico a la hora de impulsar mejoras educativas y enfrentar muchos de los nuevos retos y problemas de la educación actual. La proliferación de información, la generación de 
nuevo conocimiento y el uso de tecnologías digitales ha hecho imprescindible definir una serie de nuevas competencias para el ejercicio de la docencia. Estas competencias implican el uso de mecanismos digitales, cuyo dominio comienza con su utilización, como instrumentos para tratar, almacenar y transmitir información; pero sobre todo como medios de enseñanza y aprendizaje, lo que implica también su comprensión crítica. Es decir, no basta con saber consultar un material multimedia, sino que es necesario integrarlo con sentido en una secuencia didáctica determinada, descartarlo cuando no obedece a nuestros intereses como docentes, y crear uno nuevo (preferiblemente con los estudiantes) acorde a nuestra visión y necesidades.

En la UJAT se proporciona un ambiente flexible, dinámico y práctico que le permite al estudiante administrar su tiempo y ser el protagonista de su propio aprendizaje. El profesor guía y orienta el aprendizaje del estudiante, a través de estrategias de enseñanza y actividades para que éste adquiera las competencias señaladas en su plan de estudio, apoyándose en el uso de la plataforma tecnológica institucional a través de las aulas virtuales en donde interactúan los profesores y alumnos, brindando asesoría en línea. En cada División Académica de la universidad en donde hay Educación a Distancia hay un Tutor de Seguimiento quien es el responsable de atender y resolver asuntos académicos y tecnológicos de profesores y alumnos que se presenten durante el desarrollo de las asignaturas, si existen dudas el estudiante puede dirigirse al Tutor de Seguimiento, además del profesor.

Ante la gran necesidad de adquirir y desarrollar nuevos conocimientos, habilidades y destrezas en un mundo saturado de tecnologías digitales, parece sumamente importante el establecimiento de una buena conexión entre los programas de formación inicial y permanente con el profesorado debido a que los estudiantes que se están formando en la universidad conozcan lo que está sucediendo en el sistema educativo y por último, para que quienes tienen la gran responsabilidad de formación permanente prevean las necesidades de formación integral de quienes se integran al sistema, de esta manera el profesor se convierte en organizador, planeador y orientador siendo el estudiante el centro del proceso, con una condición mucho más autónoma, en el que las tecnologías permiten mayor motivación e independencia en la decisión de los tiempos, la autorregulación del aprendizaje entre otros elementos. Pero en el proceso de enseñanza-aprendizaje en línea, se requiere que los profesores estén más capacitados en el uso de las tecnologías tanto en los contenidos como en el dominio de la didáctica para que se propicie un mejor aprendizaje por parte de los estudiantes.

Actualmente es fundamental que el profesor adquiera habilidades, conocimientos, competencias y actitudes que lo capaciten para aplicar estrategias innovadoras y modelos alternativos, que incluyan la enseñanza por medio de TIC, en donde el alumno tenga un rol activo y una mayor responsabilidad de su aprendizaje en el proceso. Debido a esto, las instituciones de educación superior deben transformarse, adaptarse a los cambios para hacer frente a las nuevas demandas educativas de las sociedades del conocimiento. La universidad y particularmente los profesores universitarios tienen la responsabilidad de contribuir, con una práctica educativa innovadora, en la formación integral de individuos con competencias suficientes debido a que existe una gran cantidad de de información fluyendo a velocidades impresionantes a través de Internet y otros medios electrónicos, y resulta muy fácil y rápido acceder a ella y es en esta extensión de información es posible que el estudiante encuentre materiales actualizados y de mejor calidad académica que incluso el material utilizado por su profesor. Es por eso que en esta problemática la educación superior requiere de un nuevo perfil del docente universitario, el cual debe de poseer ciertos conocimientos, habilidades y actitudes para ejercer en el aula una práctica educativa innovadora y así poder estar en la dinámica de la revolución tecnológica (González, 2008).

Vidal, Rodríguez, Delgado, y Manrique (2009), aseveran que en las universidades virtuales, la formación docente permanente o continua, brinda a este profesional de perfil amplio, opciones de continuidad de estudio para su perfeccionamiento a través de cursos cortos y diplomados virtuales; el rol de los profesores, así como el plan de estudios en cuanto al desarrollo de contenidos, métodos, recursos de aprendizaje, evaluación y certificación; con un enfoque curricular sistemático, flexible, pertinente, transversal, contextualizado, integrado e investigativo, está definido en sus objetivos de enseñanza educativos, que abordan problemas según sea la modalidad presencial o virtual. 
La formación docente siempre debe de hacerse de acuerdo a los programas de estudio que se estén impartiendo y así como una en el uso de recursos tecnológicos para que éstos se puedan dar a conocerse en las aulas virtuales o presenciales, según sea el tipo de estudiante. Los tipos de estudiantes pueden ser presenciales, semi presenciales y a distancia pero esto no debe de ser una escusa para que el docente deje de prepararse ya que la educación actualmente requiere un nuevo perfil de docente universitario, que posea ciertas habilidades, conocimientos y actitudes para poder dar su clase ya sea presencial o virtual, por ejemplo en la actualidad contamos con una cantidad de información tan amplia y extensa como es la internet, en donde existen muchísimos sitios web, en donde los alumnos pueden construir sus aprendizajes y al poseer diversos recursos para comunicarnos con los estudiantes como son el correo electrónico, messenger, chats, facebook y todas las redes sociales; muchas veces los alumnos están más actualizados que el propio docente, por eso de vital importancia la capacitación del mismo. Al incorporar la tecnología en las universidades en el caso de las clases semipresenciales o virtuales ha facilitado facilitado el continuo aprendizaje y ha cambiado la forma en que se comunican los docentes con los alumnos, por lo que el uso de las TIC debe de ser un apoyo más para el profesor.

\section{Conclusiones}

El docente es un factor clave para la implementación de la tecnologías de la información y depende de su actitud ante los cambios, el éxito o fracaso para iniciar los procesos de cambio, ya que la combinación de los medios tecnológicos adecuados y un diseño didáctico basado en las necesidades específicas de aprendizaje actuales, será lo que caracterice la práctica educativa, mientras que el alumno es la parte central del aprendizaje.

La tecnología en la Universidad Juárez Autónoma de Tabasco tiene que cumplir muchos retos que en la educación, y se tienen que seguir evolucionando en las diversas formas de planear, organizar e implementar las acciones educativas dentro del contexto social, desde el punto de vista universitario, ya que no sólo es suficiente contar con recursos tecnológicos de punta sino que hay que formar buenos usuarios y consumidores de tecnología es por eso que hoy en día está abriendo campus en lugares lejanos para que todos los estudiantes tengan acceso a la educación.

Las nuevas tecnologías también están afectando a los procesos educativos generados en las universidades debido a que existe cada vez más educación fuera de la escuela en relación a la que de proporciona dentro de la escuela esto puede ser a través de soportes multimedia, de software didáctico, de televisión digital, de programas de formación a distancia, de las redes telemáticas, los mismo smartphones. Se puede afirmar que las experiencias desarrolladas por las nuevas podemos afirmar, que desde un punto de vista específicamente instructivo, es indudable que las experiencias de enseñanza desarrolladas con las nuevas tecnologías han demostrado que, son altamente motivantes para los estudiantes y son, en gran medida, eficaces en el logro de ciertos aprendizajes si las comparamos con los procesos tradicionales de enseñanza basados en la tecnología impresa. 


\section{REFERENCIAS}

Alvarado, A. y Dorrego, E. (2003). Tecnología educativa y tecnologías de la comunicación en Venezuela. Comunicar, 021, pp. 69-76.

Edel-Navarro, R.(2010). Entornos Virtuales de aprendizaje. La contribución de lo virtual en Educación. Revista Mexicana de Investigación Educativa, 15(44), pp. 7-15. Recuperado: http://www.redalyc.org/articulo.oa?id=14012513002\#.

González, J. (2008). TIC y la transformación de la práctica educativa en el contexto de las sociedades del conocimiento. RU\&SC. Revista de Universidad y Sociedad del Conocimiento [en línea], 5(2), pp. 1-8. Recuperado de http://www.uoc.edu/rusc/5/2/dt/esp/gonzalez.pdf

López, M. (2008). ¿Por qué hablar de usos educativos en las nuevas tecnologías? Revista Electrónica de Investigación Educativa, 10(2), pp. 1-6. Recuperado de http://redie.uabc.mx/ contenido/vol10no2/contenido-lopezornelas.pdf

- (2008). Reseña de "Nuevas tecnologías y educación. Diseño, desarrollo, uso y evaluación de materiales didácticos" de I. Ogalde Careaga y M. González Videgaray. REDIE. Revista Electrónica de Investigación Educativa, 10(2), pp. 1-6.

Martínez, R. y Heredia, Y. (2010). Tecnología educativa en el salón de clase. Estudio retrospectivo de su impacto en el desempeño académico de estudiantes universitarios del área de Informática. Revista Mexicana de Investigación Educativa, 15(45), pp. 371-390.

Mestre, U. y Valdés, P. (2010). Modelo de enseñanza-aprendizaje con tecnología multimedia para la modalidad de estudio semipresencial. Praxis Educativa, 16(14), pp. 35-41.

Ortega, C.F. (2010). Tecnología educativa. La formación del profesorado en la era de internet. Revista de la Educación Superior, XXXIX(156), pp. 115-118.

Piscitelli, A. (2005). Tecnologías Educativas. Una Letanía sin ton ni son. Revista de Estudios Sociales, 22, pp. 127-133.

Salas, F. (2002). Epistemología, Educación y Tecnología Educativa. Educación, 26(001), pp. 9-18.

- (2005). Hallazgos de la investigación sobre la inserción de las tecnologías de la información y la comunicación (tic) en la enseñanza: la experiencia de los últimos 10 años en los estados unidos. Educación, 29(002), pp. 53-66.

Sánchez, E. (2008). Las tecnologías de información y comunicación (tic) desde una perspectiva social. Revista Electrónica Educare, XII, pp. 155-162. Recuperado: http://www.redalyc.

Sancho, J., Ornellas, A., Sánchez, J., Alonso, C. y Bosco, A. (2008). La formación del profesorado en el uso educativo de las TIC: una aproximación desde la política educativa. Praxis Educativa, 12 , pp. 10-22. org/pdf/1941/194114584020.pdf

Tello, E. (2007). Las tecnologías de la información y comunicaciones (TIC) y la brecha digital: su impacto en la sociedad RU\&SC. Revista de Universidad y Sociedad del Conocimiento, 4(2), pp. 1-8.

Torres, A. (2005). Redes Académicas en Entornos Virtuales. Apertura, 5(001), pp. 83-91. Recuperado: http://www.anuies.mx/media/docs/89_2_1_1012161222Articulo_Angel_Torres Redes_academicas_en_entornos_virtuales.pdf

Universidad Juárez Autónoma de Tabasco [UJATa] (2013). Segundo Informe de actividades. José Manuel Piña Gutiérrez, rector. Autor. Colección Justo Sierra.

- (2013). Consulta en línea al área de Educación a Distancia. Autor, recuperado en: http://www.ujat.mx/interior.aspx?ID=229

- (2014). Sistema de Servicios Escolares. Autor.

Vidal, M. y Del Pozo, R. (2008). Tecnología educativa, medios y recursos de enseñanzaaprendizaje. Educación Médica Superior, 22(4), pp. 1-10. Recuperado de http://bvs.sld.cu/revistas/ems/vol22_4_08/ems10408.htm

Vidal, M., Llanusa, S., Diego, F. y Vialart, N. (2008). Entornos virtuales de enseñanza-aprendizaje. Educación Médica Superior, 22(1). Recuperado: http://scielo.sld.cu/scielo.php?script=sci arttext\&pid=S0864-21412008000100010 
Vidal, M., Nolla, N. y Diego F. (2009). Plataformas didácticas como tecnología educativa. Educación Médica Superior, 23(3), pp. 138-149.

Vidal, M., Rodríguez A., Delgado, A. y Manrique E. (2009). Estrategia educativa para la formación de recursos humanos en la gestión de información en salud. Revista Cubana de Salud Pública, 35(3), pp. 1-8.

\section{SOBRE LOS AUTORES}

Cecilia García Muñoz Aparicio: Licenciada en Administración de Empresas, Maestra en Administración, actualmente Coordinadora de Investigación y Posgrado y profesora investigadora de tiempo completo en la División Académica de Ciencias Económico Administrativas de la Universidad Juárez Autónoma de Tabasco; certificada por la Asociación Nacional de Facultades y Escuelas de Contaduría y Administración (ANFECA); pertenece al Sistema Estatal de Investigadores del Estado de Tabasco (SEI) en México, es perfil PROMEP (Programa del Mejoramiento al Profesorado). Secretaria de Certificación zona seis sur en México. Acreditada del Sistema Nacional de Consultores PYME por la Secretaría de Economía.

José Manuel Piña Gutiérrez: Ingeniero Agrónomo en Producción, Maestro en Administración, Doctor en Ciencias de la Administración, rector de la Universidad Juárez Autónoma de Tabasco y profesor investigador, certificado por la Asociación Nacional de Facultades y Escuelas de Contaduría y Administración (ANFECA), es perfil PRODEP (Programa Para el Desarrollo Profesional Docente) Representante del Consorcio del Golfo de Aguas Territoriales México - Estados Unidos. En el 2013 fue nombrado Presidente del Consejo Ciudadano del Premio Nacional de Periodismo, A.C.; en 2011 obtuvo el Premio Estatal de Ingeniería.

María del Carmen Ancona Alcocer: Licenciada en Administración de Empresas, Maestra en Administración, actualmente Directora y profesora investigadora de tiempo completo en la División Académica de Ciencias Económico Administrativas de la Universidad Juárez Autónoma de Tabasco; certificada por la Asociación Nacional de Facultades y Escuelas de Contaduría y Administración (ANFECA); pertenece al Sistema Estatal de Investigadores del Estado de Tabasco (SEI) en México, es perfil PRODEP (Programa Para el Desarrollo Profesional Docente). Secretaria de Certificación zona seis sur en México. Acreditada del Sistema Nacional de Consultores PYME por la Secretaría de Economía. Coordinadora Regional de Intercambio Académico en ANFECA.

María del Carmen Navarrete Torres: Licenciada en Ciencias y Técnicas de la Comunicación, Maestra en Administración, profesora investigadora de tiempo completo en la División Académica de Ciencias Económico Administrativas de la Universidad Juárez Autónoma de Tabasco; certificada por la Asociación Nacional de Facultades y Escuelas de Contaduría y Administración (ANFECA); es perfil PRODEP (Programa para el Desarrollo Profesional Docente) pertenece al Sistema Estatal de Investigadores del Estado de Tabasco (SEI) en México. Acreditada del Sistema Nacional de Consultores PYME por la Secretaría de Economía. 